\title{
Albedo and size of (99942) Apophis from polarimetric observations $\dagger$
}

\author{
Alberto Cellino ${ }^{1}$, Marco Delbò $\grave{1}^{1,2}$ and Edward F. Tedesco ${ }^{3}$ \\ ${ }^{1}$ INAF - Osservatorio Astronomico di Torino, \\ strada Osservatorio 20, 10025 Pino Torinese, Italy \\ email: cellino@inaf.oato.it \\ ${ }^{2}$ Observatoire de la Côte d'Azur, BP 229, Nice, France \\ email: delbo@obs-nice.fr \\ ${ }^{3}$ University of New Hampshire, USA \\ email: Ed.Tedesco@unh.edu
}

\begin{abstract}
We have obtained the first accurate determination of the albedo of (99942) Apophis, by means of polarimetric observations carried out at the VLT. The observations allowed us to obtain the slope of the polarization-phase curve of this object, from which an albedo estimate of $0.33 \pm 0.04$ could be obtained. From our observations we also obtained a new estimate of the absolute magnitude: $H=19.7 \pm 0.2$ (assuming $\mathrm{G}=0.25$, which applies to $\mathrm{S}$ - and Q-type asteroids). Based on these results, we derive for the size of Apophis a value of $270 \pm 30$ meters. The accuracy of this size estimate is mostly related to uncertainties in $H$, whereas the obtained albedo value should be considered more robust. Our observations convincingly show that polarimetry is an effective and efficient tool to obtain accurate albedos and sizes for small and faint potentially hazardous asteroids.
\end{abstract}

Keywords. Asteroids, polarization

\section{Introduction}

The near-Earth object (99942) Apophis will make an extremely close approach to the Earth in 2029, possibly followed by resonant returns starting in 2036. The computation of the orbital evolution of near-Earth asteroids (NEAs), including potentially hazardous asteroids (PHAs) like Apophis, is currently limited by insufficient knowledge of some physical properties of the objects, required to compute the orbital drift produced by the Yarkovsky effect (Bottke et al. 2002). Moreover, knowledge of the size of the objects is needed also to estimate the amount of kinetic energy delivered to the Earth in the case of a collision with our planet. For these reasons, the exploitation of observing techniques which can provide reliable and accurate estimates of the basic physical properties of NEAs, including their sizes, is a high-priority task.

We present here the first determination of the albedo of (99942) Apophis, obtained by means of polarimetric observations carried out at the Very Large Telescope of the European Southern Observatory at Cerro Paranal (Chile). The observations allowed us to obtain the slope of the polarization - phase curve of Apophis, from which we derived the albedo of the object. Our observations also allowed us to obtain a new estimate of the absolute magnitude of Apophis. Based on these results, we use the well known relation relating albedo, absolute magnitude and size, to derive the equivalent diameter of this asteroid.

$\dagger$ Based on observations obtained at the European Southern Observatory (ESO), DDT request 276.C-5030 
We note that a detailed description of our VLT observations and a thorough discussion of the obtained results is given in a separate paper (Delbò, Cellino \& Tedesco 2006). Here, we only briefly summarize the results of our investigation, and briefly mention the potential role that polarimetry can play in the future for the purposes of physical characterization of NEAs and PHAs.

\section{Asteroid Polarimetry}

It is known that polarimetry is among the best available tools that may be adopted to derive asteroid albedos. Here, we briefly summarize the fundamental background of this technique. The light that we receive from asteroids at visible wavelengths consists of sunlight scattered by the solid surface of the bodies, and is therefore in a state of partial linear polarization. The degree of linear polarization varies for changing illumination conditions in a characteristic way, and can be described by plotting it as a function of the phase angle, namely the angle between the directions of the Earth and the Sun as seen from the asteroid. More precisely, the state of linear polarization is a vector, characterized by a module, (given by the square root of the sum of the squares of the Stokes parameters $P_{x}$ and $P_{y}$ which characterize the state of linear polarization of the received light) and a direction, represented by a Position Angle (given by $\arctan \left(P_{y} / P_{x}\right)$ ) which gives the orientation of the plane of polarization with respect to a reference direction. In the case of polarimetric measurements of atmosphereless solar system bodies like the asteroids, it is found that the plane of linear polarization is in general either parallel or perpendicular to the plane of scattering, defined as the plane containing the Sun, the target and the observer at the epoch of observations. For this reason, the state of linear polarization of the asteroids is commonly described by means of the parameter $P_{r}=\frac{\left(I_{\perp}-I_{\|}\right)}{\left(I_{\perp}+I_{\|}\right)}$. In the above definition $I_{\perp}$ and $I_{\|}$indicate the intensity of the asteroid light having the plane of polarization perpendicular and parallel to the scattering plane, respectively (Dollfus \& Zellner 1979, Dollfus et al. 1989).

When one plots the variation of $P_{r}$ as a function of the phase angle, a well defined curve is usually obtained. A typical example is shown in figure 1. Phase-polarization curves are always characterized by the presence of a range of phase angles, usually between $0^{\circ}$ and $20^{\circ}$, for which $P_{r}$ is negative and reaches a minimum at a phase angle around $10^{\circ}$. This general trend characterizes, with some minor differences depending on the taxonomic class, all asteroids observed so far. Beyond a phase angle of about $20^{\circ}$ (inversion angle), the polarization usually changes sign, and becomes positive. Around and beyond the inversion angle, the trend of Pr for increasing phase angle is essentially linear, and a well defined relation is known to exist between the slope of the linear part of the polarization curve and the albedo of the surfaces. This is the so-called slope-albedo law, and takes the form $\log p_{V}=C_{1} \log h+C_{2}$, where $p_{V}$ is the albedo, and $h$ the polarization slope. The most recent derivation of the $C_{1}$ and $C_{2}$ coefficients has been published by Cellino et al. (1999).

\section{Observations and results}

In our 2006 observing campaign we observed (99942) Apophis in $V$ light on four nights, on February 16, March 7, March 28 and April 2, respectively. The phase angles at the four epochs of observation were about $64^{\circ}, 68^{\circ}, 79^{\circ}$ and $83^{\circ}$, respectively. The $P_{r}$ parameter was measured on each night, and from this we could derive a value of the slope equal to $0.069 \pm 0.004$. The results of our polarimetric measurements are graphically shown in the quoted Delbò, Cellino \& Tedesco (2006) paper. 


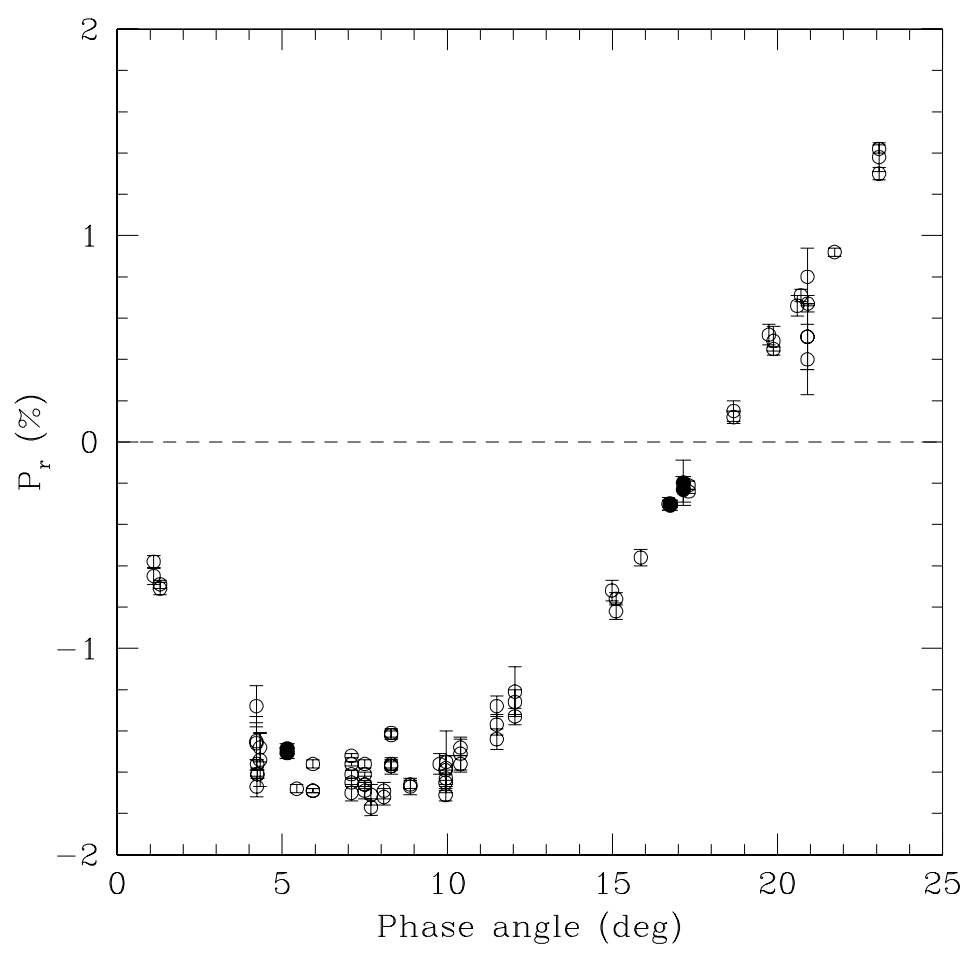

Figure 1. Phase-polarization curve of the asteroid (1) Ceres. Different symbols indicate measurements taken from different data-sets available in the literature.

Using the Cellino et al. (1999) calibration, and taking into account the nominal uncertainties both in the calibration coefficients and our measured polarimetric slope (the latter being a function of the error bars of our single polarimetric measurements), we derived an albedo value of $0.33 \pm 0.04$.

For each of our observations, we derived also some accurate estimates of the apparent magnitude of our target. From these photometric measurements, we could obtain four points of the phase - magnitude curve of (99942) Apophis during the 2006 apparition. These measurements can be used to extrapolate the magnitude of the object at zero phase angle, to derive its absolute magnitude, namely the magnitude at zero phase angle, as would be measured by an observer looking at the asteroid when it is at a distance of $1 \mathrm{AU}$ from both the Sun and the observer. It is usual to indicate by means of the $H$ symbol the absolute magnitude in $V$ light, and to use another parameter, called $G$, to describe the non-linear variation of the magnitude as a function of phase angle, including the so-called "opposition effect", namely a non linear brightness surge at phase angles of a few degrees (Bowell et al. 1989). The $(H, G)$ system has been officially adopted by the IAU Commission 20 in 1985. As mentioned above, our observations cover only four nights and were obtained at quite large phase angles, then they can hardly be used to derive a simultaneous solution for both the $H$ and $G$ parameters. For this reason, we computed best-fit values for the $H$ parameter only, making some a priori assumptions on the value of $G$. By choosing for $G$ the value of 0.25 , which is the value commonly found for $S$ and $Q$-type asteroids, we derive for $H$ a value of $19.7 \pm 0.1$. Assuming for $G$ the typical default value of 0.15 , gives correspondingly $H=19.5 \pm 0.1$. Given the uncertainties in the choice of $G$, we assume the following value for the absolute magnitude of (99942) 
Apophis: $H=19.7 \pm 0.2$. Note that this is our choice based on the data we have at the moment of submitting this paper within the deadline for publication. We cannot rule out that in the final version of the Delbò, Cellino \& Tedesco (2006) paper, which will present the definitive results of this analysis, we might adopt a slightly different value.

From a knowledge of the albedo and the absolute magnitude, we can finally derive an equivalent size of $270 \pm 30$ meters for Apophis. This value, is slightly but not negligibly smaller than the value previously assumed and reported in the NASA web page http://neo.jpl.nasa.gov/risk, namely 320 meters.

We note that our investigation has led to an albedo and size measurement for the smallest asteroid ever observed using the polarimetric technique. In particular, the most robust result of our investigation is certainly the albedo of (99942) Apophis, since this is the parameter that can be derived most directly from the polarimetric measurements. The size is more uncertain, due to the quoted uncertainties in our derived value of the absolute magnitude, which suffers from a poor knowledge of the $G$ parameter. A more accurate derivation of the size will be possible in the future, when new observations of this asteroid will produce a more accurate determination of its absolute magnitude.

The present results show that the availability of large instruments equipped with modern CCD polarimeters can be a major breakthrough in the field of the physical characterization of NEAs and PHAs.

\section{Acknowledgements}

We thank the the staff and the Science Archive Operation of the ESO for their support to our observations. The work of Marco Delbò was partially supported by the European Space Agency (ESA) and that of E.F. Tedesco by the National Aeronautics and Space Administration (NASA) under grant NNG04GK46G, issued through the Office od Space Science Research and Analysis Programme.

\section{References}

Bottke, W.F., Vokrouhlický, D., Rubincam, D.P., \& Brož, M. 2002 in: W.F. Bottke, A. Cellino, P. Paolicchi, R.P. Binzel, (eds.), Asteroids III (University of Arizona Press), p. 395

Bowell, E., Hapke, B., Domingue, D., Lumme, K., Peltoniemi, J., \& Harris, A.W. 1989 in: R.P. Binzel, T. Gehrels, M.S. Matthews, (eds.), Asteroids II (University of Arizona Press), p. 524

Cellino, A., Gil-Hutton, R., Tedesco E.F., Di Martino, M., \& Brunini, A. 1999, Icarus 138, 129

Delbò, M., Cellino, A. \& Tedesco E.F. 2006, Icarus, submitted

Dollfus, A. \& Zellner, B. 1979 in: T. Gehrels, (ed.), Asteroids (University of Arizona Press), p. 170

Dollfus, A., Wolff, M., Geake, J.E., Lupishko, D.F., \& Dougherty, L. 1989 in: R.P. Binzel, T. Gehrels, M.S. Matthews, (eds.), Asteroids II (University of Arizona Press), p. 594 